\title{
EFFECTS OF FILTERING ON BER PERFORMANCE OF AN OFDM SYSTEM
}

\author{
Navjot Kaur ${ }^{1}$, V.K. Banga ${ }^{2}$ \\ ${ }^{1}$ M.Tech.Scholar, ${ }^{2}$ Principal, A.C.E.T. College, Punjab, India, navjosan2011@gmail.com,vijaykumar.banga@gmail.com
}

\begin{abstract}
In upcoming generations, wireless communication system requires a higher standard in order to provide high quality of services to customers. Orthogonal Frequency Division Multiplexing (OFDM) is an efficient modulation technique of forthcoming wireless systems which can be implemented easily. In this paper, an effort has been made to analyze how well an OFDM system can perform when a signal is transmitted over an Additive White Gaussian Noise (AWGN) channel using 16 QAM modulation techniques. The performance of OFDM system with pulse shaping filters are also evaluated and results reveal that the pulse shaped OFDM improve the overall performance of the system in terms of BER. We use Different pulse shaping filters such asRaised cosine, FIR Nyquist and $S Q R T$ Raised Cosine for analyzingthe Bit Error Rate (BER) performance.
\end{abstract}

Index Terms: AWGN, ICI, OFDM, Bit Error Rate.

\section{INTRODUCTION}

High bit error rate(BER) and high bandwidth efficiency are the major desiresfor modern communication systems to satisfy the needs for a variety of new and high quality services developed and delivered to the society [1]. The effect of multipath delay, spread and fading of signals in the wireless environment is usually unavoidable [2], [3]. Extreme fading of the signal amplitude and Inter Symbol Interference (ISI) introduced during transmission through the conventional channel and frequency selectivity of the channel appearing at the receiver side [4], [5] are responsible for a high probability of errors and reduced overall performance of the system. Several methods like adaptive equalization and channel coding have been developed to reduce the above effect. [6], [7]. The orthogonal Frequency division multiplexing (OFDM) a multi carrier system can be thought of as a solution and is used in several applications such as asymmetric digital subscriber lines (ADSL), a system that makes high bit-rates possible over twisted-pair copper wires [8], [9], it has also been accepted for several wireless LAN standards, as well as a number of mobile multimedia applications [1], [10] and protocols like IEEE 802.11a standard, terrestrial digital video broadcasting (DVB$\mathrm{T})$ and digital audio broadcasting (DAB) [11]. The purpose of this paper is to investigate how OFDM performs in an Additive White Gaussian Noise (AWGN) channel for different pulse shaping filter like Raised cosine, FIR Nyquist and Matched SQRT Raised Cosine. In this channel there is only one path between transmitter and receiver and a constant attenuation and noise is considered. In this paper, we define the simulation methodology of OFDM in section 2 and simulate the OFDM system using Matlab. The mathematical model and theoretically simulated constellation diagrams for various pulse shapes are presented and a procedure for symbol error rate is derived and finally a BER simulation of OFDM for various pulse shaping filters at different SNR value is presented.

\section{SYSTEM MODEL}

Figure 1(a) and 1(b) show the structure of a typicalOFDM communication system with various pulse shaping filters. In this communication system, QAMconstellation is used to map binary information. The high speed serial data stream is split up into a set of low speed sub streams and modulated onto the orthogonal carriers through Inverse Fast Fourier transform (IFFT) [2].

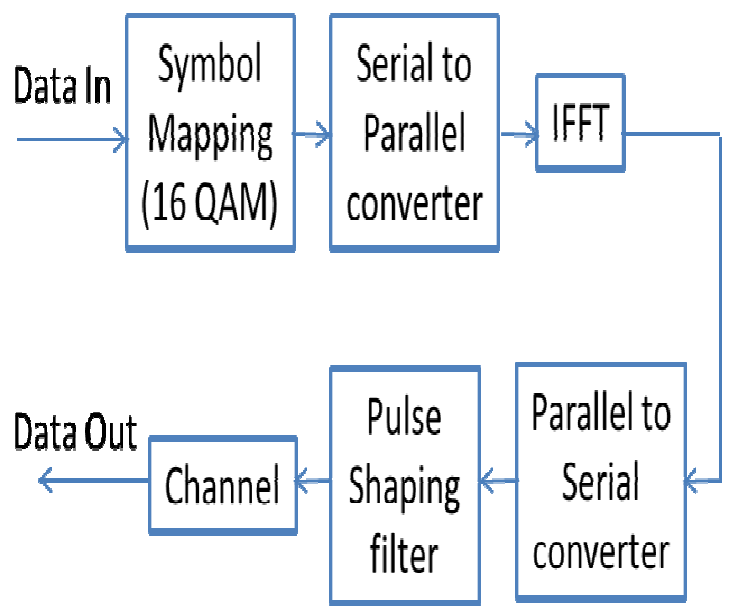

Figure-1(a): Transmitter of a pulse shaped OFDM system 




Figure-1(b): Receiver of a pulse shaped OFDM System

OFDM with pulse-shaping filter can be representedas:

$$
s(t)=e^{j 2 \pi f_{c} t} \sum_{k=0}^{N-1} D_{k} p(t) e^{j 2 \pi f_{k} t}
$$

Where $\mathrm{j}=\sqrt{-1}$, Number of subcarrier is $\mathrm{N}$, Carrier frequency of OFDM system isf $f_{c}$, Subcarrier frequency of kth subcarrier is, $\mathrm{f}_{\mathrm{k}}$ and here $\mathrm{k}=0,1 \ldots \mathrm{N}-1$, and $\mathrm{p}(\mathrm{t})$ is time limited pulse shaping filter. Transmitted symbol is Dk which is assumed to have zero mean and normalized average symbol energy. Also we consider that all data symbols are uncorrelated $[9,10]$ i.e.

$$
\mathrm{E}\left[\mathrm{D}_{\mathrm{k}} \mathrm{D}_{\mathrm{m}}^{*}\right]= \begin{cases}1, & \mathrm{k}=\mathrm{m} \\ 0, & \mathrm{k} \neq \mathrm{m}\end{cases}
$$

where $\mathrm{m}=0,1, \ldots \ldots, \mathrm{N}-1$

Where $\mathrm{D}_{\mathrm{m}}^{*}$ is the complex conjugate of $\mathrm{Dk}$.To ensure the orthogonality of subcarrier, it is very important to satisfied the below equation for OFDM system [9, 10]. The subcarrier frequency is

$$
\begin{aligned}
\mathrm{f}_{\mathrm{k}} & =\frac{\mathrm{k}}{\mathrm{T}_{\mathrm{s}}} \ldots(2.3) \\
\text { wherek } & =0,1, \ldots \ldots \ldots, \mathrm{N}-1 \\
\mathrm{f}_{\mathrm{k}}-\mathrm{f}_{\mathrm{m}} & =\frac{\mathrm{k}-\mathrm{m}}{\mathrm{T}_{\mathrm{s}}} \ldots(2.4)
\end{aligned}
$$

where $\mathrm{k}=\mathrm{m}=0,1, \ldots \ldots, \mathrm{N}-1$

For maintaining orthogonality between subcarriers, the minimum required subcarrier frequency spacing is $1 / \mathrm{T}_{\mathrm{s}}$. The received signal at the receiver can be represented as:

$$
r(t)=s(t) \otimes h(t)+w(t) \ldots
$$

In above equation convolution is denoted by $\otimes, \mathrm{h}(\mathrm{t})$ is the channel impulse response and the additive white Gaussian noise is represented by $w(t)$ which process with zero mean and variance $\mathrm{NO} / 2$ per dimension.

\section{PULSE SHAPING FILTER}

Each carrier in the OFDM spectrum is represented by main lobe with a number of side lobes having lower amplitudes. Since peak power is associated with main lobe and ICI power is associated with side lobes, so the motive of pulse shaping filter is to increase the width of main lobe and/or reduce the amplitude of side lobes [9]. Pulse shaping technique is used to transmit data within a limited bandwidthwhich further reduces the Inter Symbol Interference(ISI) [11]. In this section some most commonly used pulse shaping filters have been introduced. These pulse shaping filters are Raised Cosine,FIR Nyquist and Square Root Raised Cosine Filter. ICI power for various shaping pulses is:

a) Raised Cosine Pulse

$$
P_{R C}(f)=\operatorname{sinc}(f t) \frac{\cos (\pi \alpha f t)}{1-(2 \alpha f t)^{2}}
$$

Where ' $\alpha$ ' is roll-off factor $(0 \leq \alpha \leq 1)$ and $\mathrm{f}$, $\mathrm{t}$ are called frequency $\&$ time respectively.

b) Square Root Raised Cosine Pulse

$$
\begin{aligned}
P_{\text {srrc }}(f)= & \operatorname{sinc}(f t)\left(\frac{4 a}{\pi \sqrt{f}} \cos (1+a) \frac{\pi f}{t}\right)+ \\
& \frac{\left(\frac{t}{4 a f} \sin (1-a) \frac{\pi a}{t}\right)}{\left(1-\left(\frac{4 a f}{t}\right)^{2}\right)} \ldots . .(3.2)
\end{aligned}
$$

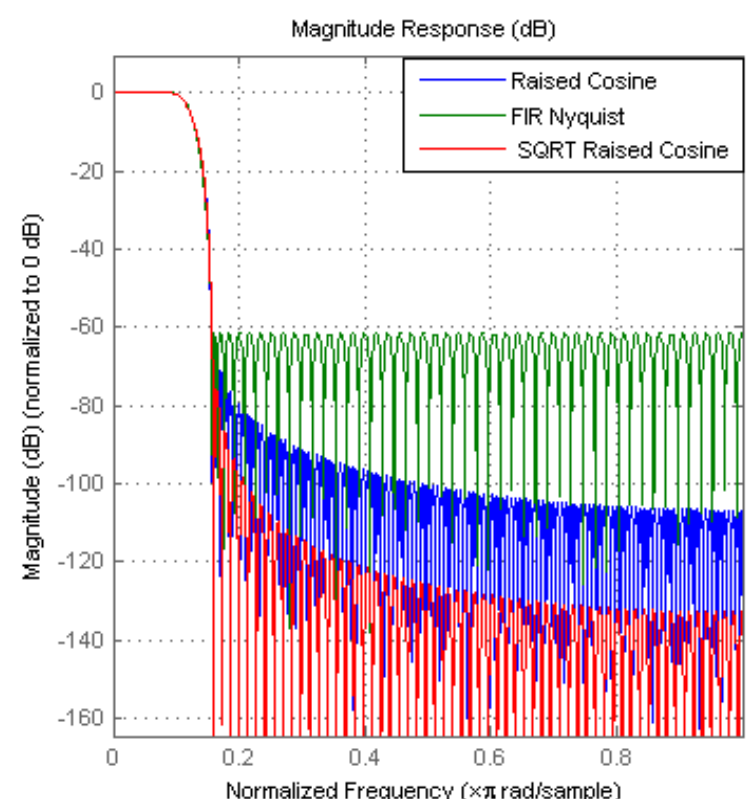

Figure-2: Magnitude Response of various filters 


\section{SIMULATION RESULTS}

Simulation results have been prepared to demonstrate the performance enhancement of shaping an OFDM system using various pulse shaping filters which have been mentioned in section 3 .

Table-1: BER of OFDM communication system under various filters with varying SNR

\begin{tabular}{|l|l|l|l|l|}
\hline & $\begin{array}{l}0-\mathrm{dB} \\
\text { SNR }\end{array}$ & $\begin{array}{l}\text { 04-dB } \\
\text { SNR }\end{array}$ & $\begin{array}{l}\text { 08-dB } \\
\text { SNR }\end{array}$ & $\begin{array}{l}\text { 12-dB } \\
\text { SNR }\end{array}$ \\
\hline $\begin{array}{l}\text { Simple } \\
\text { OFDM }\end{array}$ & 0.2956 & 0.2129 & 0.1011 & 0.0209 \\
\hline $\begin{array}{l}\text { RC- } \\
\text { OFDM }\end{array}$ & 0.2845 & 0.2045 & 0.0887 & 0.0184 \\
\hline $\begin{array}{l}\text { FIR- } \\
\text { Nyquist } \\
\text { OFDM }\end{array}$ & 0.2875 & 0.2041 & 0.0967 & 0.0196 \\
\hline $\begin{array}{l}\text { SRRC- } \\
\text { OFDM }\end{array}$ & 0.0764 & 0.0110 & 0.0001 & 0 \\
\hline
\end{tabular}

Some outcomes have been derived on thebasis of Bit Error Rate (BER) and Impulse Response. In this experimental test what we come to know is, matched square root raised cosine pulse shaped OFDM has outperformed.

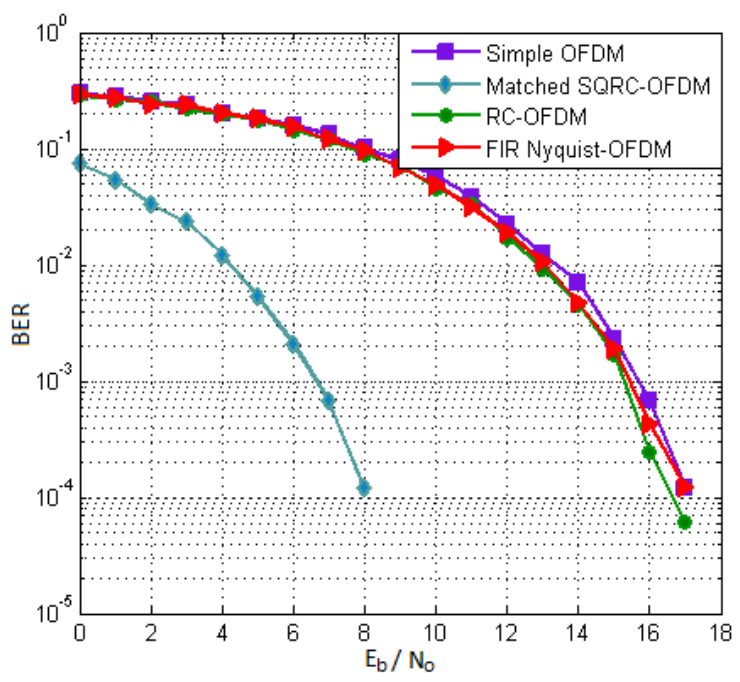

Figure-3: BER of OFDM with or without Pulse Shaping Filter

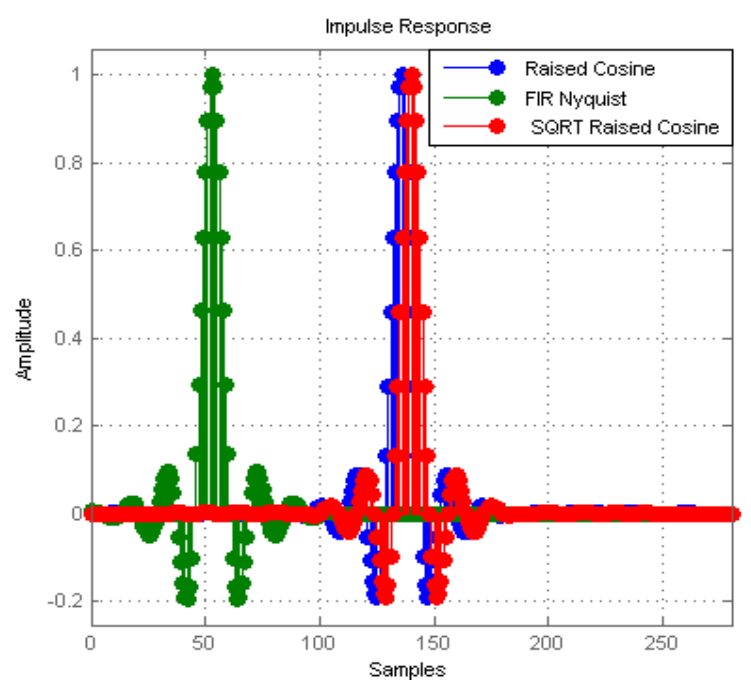

Figure-4: Impulse Response of OFDM with different Pulse Shaping Filter

\section{CONCLUSIONS}

In this work, simulation results have been presented to demonstrate that pulse shaped OFDM significantly enhances the performance of communication system as compared to simple OFDM. It can be concluded that if pulse shaping filter has the spectrum with higher width of main lobe and smaller amplitude of side lobes, then pulse shaping filter will lead less ICI and better bandwidth efficiency.

In this paper, we have studied and analyzed the family of pulse shaping filters. We have evaluated the performance of these pulse shaping filters with OFDM in terms of BER with varying SNR values for 16-QAM modulation under AWGN channel. From this BER analysis it can be concluded that performance of pulse shaped OFDM is better as compared to simple OFDM. Simulation results reveal that pulse shaped OFDMwith Square Root Raised Cosine filterdesign method has less BER as compared to others so it can be a better option in contrast to other pulse shaping filter.

\section{REFERENCES}

[1]. S. D. Assimonis et. al., "OptimizedBetter than RaisedCosine Pulse for Reduced ICI in OFDM Systems”, 17th International Conference on Telecommunications, 2010.

[2]. M.Palaivelan, S. Anand and S. P. Venkatesan, "PAPR and ICI Reduction in OFDM Systems using Modified Raised Cosine Power Pulse Shape", European Journal of Scientific Research, ISSN: 1450-216, Vol.72, No.4, pp. 618-627, 2012.

[3]. Srabani Mohapatra and Susmita Das,"A New Approach for Performance Improvement of OFDM System Using Pulse Shaping", NIT Rourkela, 2009. 
[4]. S. Aenagandula, A. Kumar, Srinivas K. and M. Nanda, “ Inter Carrier Interference and Signal to Interference Ratio of various Pulse Shaping Filters used in OFDM System with Carrier Frequency Offset”, IJESS (International Journal of Electronics Signals and Systems), ISSN: 2231-5969, Vol-1, Iss-3, 2012.

[5]. Ali T. Shaheen, "Design and Simulation of Hartley based Multi Orthogonal Band OFDM", International Journal of Computer Applications, Volume 68, Issue 15, pp. 10-16, April 2013.

[6]. J. Armstrong, "Analysis of new and existing methods of reducing inter-carrier interference due to carrier frequency offset in OFDM," IEEE Transactions on Communications, Vol. 47, No. 3, pp. 365-369, March 1999.

[7]. H.M. Mourad, "Reducing ICI in OFDM systems using a proposed pulse shape", Wireless Person. Communications, vol. 40, pp: 41-48, 2006.

[8]. Volkan Kumbasar et al,"ICI reduction in OFDM systems by using improved sinc power pulse", Digital Signal ProcessingAcademic Press, Inc. Orlando, FL, USA, vol. 17, issue 6, pp. 997-1006, November 2007.

[9]. Srabani Mohapatra and Susmita Das, "Performance Enhancement of OFDM System with ICI Reduction Technique", In Proceedings of the World Congress on Engineering, Vol. 1, July 2009.

[10]. Nadieh M. Moghaddam and Mohammad Mohebbi, "ICI Reduction Methods in OFDM Systems", Recent Advance in Wireless Communication and Networks, Prof. Jia-Chin Lin (Ed.), ISBN: 978-953-307-274-6, August 23, 2011.

[11]. P. Tan, N.C. Beaulieu, "Reduced ICI in OFDM systems using the better than raised-cosine pulse", IEEE Commun. Lett. volume 8 issue 3, pp. 135-137, 2004.

[12]. Mahua Pal, "Algorithm for sharpening Raised Cosine Pulse shaping Digital Filter and Analysis of Performance Of QAM system when subjected to Sharpened Raised Cosine Filter", International Journal of Scientific and Research (IJSAR) Publications, Volume 2, Issue 1, ISSN 2250-3153, January 2012. 